\title{
Psychological Science Accelerator Data Management Bylaws
}

\section{Data Management Working Group:}

Patrick S. Forscher, Balazs Aczel, Ben Jones, Chris Chartier, Erica Musser, Kai Horstmann, Jon Grahe

Reviewed and suggested edits:

Zoltan Kekecs, Katherine S. Corker, Miroslav Sirota, Leigh Ann Vaughn, Aaron L. Wichman, Marietta Papadatou-Pastou, Peter Szecsi, Thomas Rhys Evans, Daniel Storage, Gerit Pfuhl, John Borghi, Heather Urry, Peder M. Isager, Hannah Moshontz, Hans IJzerman, Jessica Kay Flake

Revision history:

2019-08-20: The following sections were approved by the D\&M Committee, following minor revisions: "License"

2019-03-18: The following sections were approved by the D\&M Committee, following minor revisions: "Data Release"

2019-02-09: The following sections were approved by the D\&M Committee: "General Overview", "The Data Management Plan", "Data Repository", "Meta-Data", and "Data Integrity Checks" 


\section{Table of Contents}

$\begin{array}{ll}\text { General Overview } & 3\end{array}$

$\begin{array}{ll}\text { The Data Management Plan } & 5\end{array}$

$\begin{array}{ll}\text { Dataset security/backup considerations } & 5\end{array}$

$\begin{array}{ll}\text { Dataset structure } & 6\end{array}$

$\begin{array}{ll}\text { Codebooks } & 8\end{array}$

Confidentiality issues $\quad 9$

$\begin{array}{ll}\text { Data Repository } & 10\end{array}$

Recommended Repository Structure 11

$\begin{array}{ll}\text { Nomenclature } & 12\end{array}$

OSF project template $\quad 12$

Access settings $\quad 12$

$\begin{array}{ll}\text { Meta-Data } & 13\end{array}$

Data Integrity Checks $\quad 15$

$\begin{array}{ll}\text { Issues flagged during data collection } & 15\end{array}$

$\begin{array}{ll}\text { Checks during data processing } & 15\end{array}$

Exclusion of observations from analysis 16

$\begin{array}{ll}\text { Data Release } & 17\end{array}$

$\begin{array}{ll}\text { License } & 17\end{array}$

$\begin{array}{ll}\text { Full Release } & 18\end{array}$

$\begin{array}{ll}\text { Phased Release } & 18\end{array}$

$\begin{array}{ll}\text { Announcement of Data Release } & 18\end{array}$

Release of the Exploratory Segment $\quad 19$

Release of the Confirmatory Segment 19

$\begin{array}{ll}\text { References } & 20\end{array}$ 


\section{General Overview}

Our data management process is guided by the following principles (see also the FAIR principles; Wilkinson et al., 2016):

- Ethical data use

- Security of data storage

- Accuracy

- Usability

- Transparency

Our data management process starts immediately after the Psychological Science Accelerator (PSA) accepts a proposal (see Figure 1). First, the proposing team completes a data management plan, a document that outlines confidentiality protections, precautions to backup and secure data during collection, and data structure. The Data and Methods Committee reviews this data management plan for concerns related to our core principles of ethics, security, accuracy, usability, and transparency and requests revisions if needed.

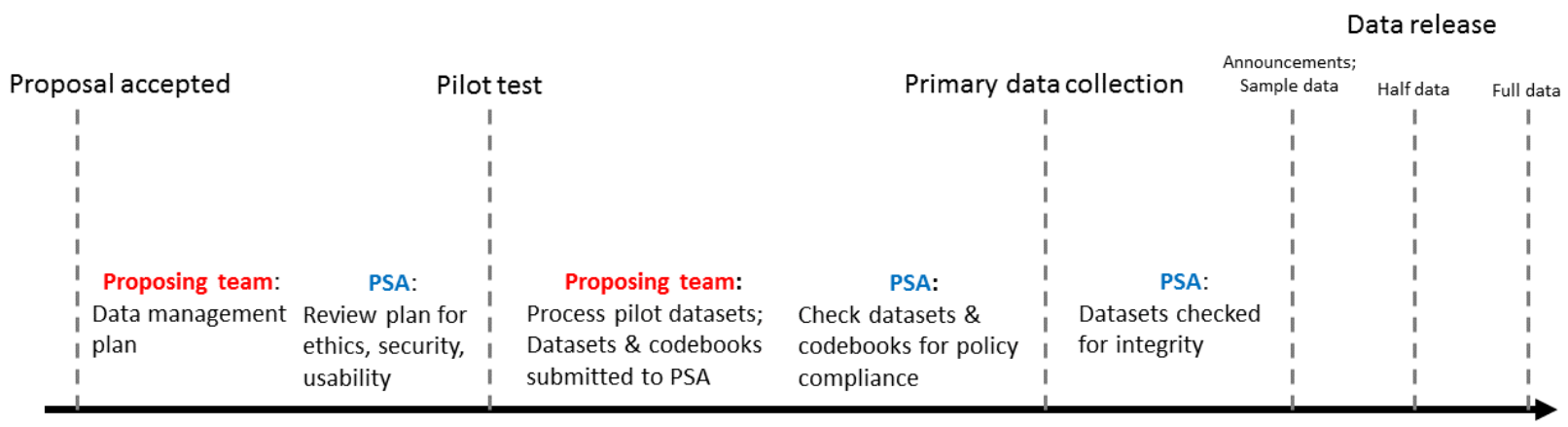

Figure 1. The Psychological Science Accelerator's data management process.

We strongly recommend that all PSA projects undergo a pilot test to catch and address potential problems with the project protocol. During the pilot test, a small number of PSA labs collect data (either with naive or non-naive participants) using the protocol prepared by the proposing team. Upon completion of the pilot test, the proposing team will process the datasets according to their data management plan (if needed) and prepare codebooks for the datasets. They should then upload these documents, along with their raw data and processed data, to an appropriate PSA-approved archive (usually, an OSF component) and provide a link to the Data and Methods Committee for review. If needed, the Data Management Committee will request modifications to the data management process. Any datasets and codebooks generated during the pilot must pass D\&M review before primary data collection occurs.

At some point after primary data collection has begun, the proposing team - with consultation from the Data and Methods Committee as needed - will provide a sample dataset so that researchers who wish to conduct secondary analyses have access to a similarly-structured 
dataset as they are writing and testing analysis code. This sample dataset could contain data collected in the pilot or the primary data collection or a previous study, but it could also be simulated or otherwise artificially generated. Regardless of how the data are generated, the sample dataset will follow the structure, formatting, and conventions established in the data management plan.

After all the sites have finished full data collection, the Data and Methods Committee will perform a data integrity check on the full dataset and correct any errors that are uncovered during these checks. After the datasets are processed, the Committee and proposing team will decide whether the data should follow an immediate or phased release process and set dates for the dataset release.

Our data management policies were written to be compliant with the Psych-DS dataset specification format, a common standard for formatting psychology datasets. As of 2019-02-05, Psych-DS is an unfinished project and the specification may evolve. We will revise this section of our Data Management Bylaws to maintain compliance with Psych-DS. 


\section{The Data Management Plan}

To complete a Data Management Plan, the proposing lab should complete this form. The proposing team's data management plan must contain the following elements:

- Dataset security/backup considerations

- An overview of the structure of the raw and processed datasets

- Codebooks for the raw and processed datasets

- Confidentiality issues and protections

\section{Dataset security/backup considerations}

During data collection, datasets should be secured so as to guard against the possibility that data are corrupted, lost, or interfered with during collection.

If data collection is centralized on a remote server, access should be password protected, and the proposing team should plan to backup the data in at least one separate location at regular intervals. If data collection is not centralized, the proposing lab should create a default plan for how data are secured. If a participating lab cannot follow this default plan due to local constraints, they will work out an alternative procedure with the proposing team. These deviations will be reviewed by the Data Management Committee to ensure that data are still properly secured.

We strongly encourage proposing teams to suggest solutions that allow for version control of the dataset. In version controlled databases, changes to the database are tracked by keeping a record of each change, the date and time of the change, and the user responsible for the change. Version control can be used to assist with correcting errors and tracking down the source of any errors that do occur. Version control also helps provide assurances as to the provenance of the data, because it can be used to demonstrate that data were not inappropriately altered during data processing or analysis. Many data repositories, such as OSF, use version control; you should consult the documentation for your repository for more information on its version control standards.

Because centralized data collection methods generally provide more streamlined backup, security, and version control processes, we encourage proposing teams to use centralized data collection methods. 


\section{Dataset structure}

The data management plan should describe the structure of any source, raw and processed datasets. A source dataset is one that is as close as possible to what was recorded during data collection - for example, scans of handwritten questionnaires. Raw data are source data that contain some minimal amount of processing - for example, spreadsheet versions of scanned questionnaires. A processed dataset is one that has been checked for integrity, corrected for errors that occur during data collection, and formatted for easy analysis. We give more details on source, raw, and processed data below.

Source datasets should contain as little processing as possible, even if, as a result of the minimal processing, direct analysis of source datasets is infeasible. Due to confidentiality and practical considerations, source datasets may not always be publicly available. For example, a set of videos that are coded for special characteristics may serve as a source dataset for a given project. However, participants who appear in videos are often readily identifiable, so unrestricted access to video source data serves as a threat to participant confidentiality. Other examples of source data include scans of handwritten questionnaires, the datasets saved by specialized data acquisition software such as Qualtrics or EEGLab, and scans of documents used for archival analysis.

Raw datasets are different from source datasets in that they are subjected to the minimum amount of processing required to transform them into tabular format. For example, a raw dataset constructed from scans of handwritten questionnaires might consist of the results of a table of questionnaire responses transcribed using Excel or other data entry software. Raw datasets should be provided in both tab-separated format (.tsv, for compliance with the PsychDS specification) and comma-separated format (.csv, for usability by people who haven't worked with tab-separated data).

In some projects, the source and raw datasets will be the same because no processing is required to transform the source dataset into tabular format. This could happen, for example, because the data acquisition software already saves acquired data as a table. Projects that do require source data to be transformed into raw data should use a reliable and reproducible process to do this transformation. The transformation need not always use computerized processes - for example, in a project with video source data, researchers could use human coders to extract variables from these videos. However, if the process used to create the raw datasets from the source datasets involves human elements, the researchers should include checks on the reliability of those human elements (e.g., through the calculation of inter-rater reliability).

Raw datasets should only be subjected to as much processing as is necessary to bring the source dataset into tabular format. Any extra processing should only occur in the processed datasets, which are the versions of the project data that are subjected to data integrity checks and that are formatted for easy analysis. As with the source $\rightarrow$ raw process, the raw $\rightarrow$ processed process should be as reliable and reproducible as possible. Unlike the source $\rightarrow$ raw process, 
where we allow human elements, we strongly prefer that the raw $\rightarrow$ processed process occur primarily within in a well-documented program or script that is archived alongside the raw and processed datasets. We also strongly prefer that the script or program that does this transformation rely on open source software like $\mathrm{R}$ rather than proprietary software so that it can be checked and used without needing to purchase proprietary software. Finally, processed datasets should be provided in both tab-separated format (.tsv, for compliance with the PsychDS specification) and comma-separated format (.csv, for usability by people who haven't worked with tab-separated data).

Processed datasets should generally be formatted according to tidy principles (Wickham, 2014): Each element in the dataset should represent a value (a number or string), each column a variable (a set of values measuring the same underlying attribute), and each row an observation (a set of values that belong to a particular unit). See Tables 1-3.

\begin{tabular}{l|c|c}
\multicolumn{1}{c|}{ name } & trt & result \\
\hline John Smith & a & -- \\
\hline John Smith & b & 2 \\
\hline Jane Doe & a & 16 \\
\hline Jane Doe & b & 11 \\
\hline Mary Johnson & a & 3 \\
\hline Mary Johnson & b & 1
\end{tabular}

Table 1. A tidy dataset, reproduced from Wickham (2014). Each column represents a discrete variable, and each row represents a single observation. Note that, because the observation represents a person's values under a specific treatment status, each person has two rows associated with their data rather than one.

\begin{tabular}{c|c} 
treatmenta & treatmentb \\
\hline-- & 2 \\
\hline 16 & 11 \\
\hline 3 & 1
\end{tabular}

Table 2. An untidy version of the dataset in Table 1, reproduced from Wickham (2014). A variable, treatment status, is stored across two column names instead of taking explicit values in the dataset. 


\begin{tabular}{c|c|c|c} 
& John Smith & Jane Doe & Mary Johnson \\
\hline treatmenta & -- & 16 & 2 \\
\hline treatmentb & 2 & 11 & 1
\end{tabular}

Table 3. An untidy dataset version of the dataset in Table 1, reproduced from Wickham (2014). A variable, person name, is stored across three column names instead of taking explicit values in the dataset.

Data often have a complex structure, with different variables applying to different units of analysis. For example, studies that follow participants over time sometimes contain data that are characteristics of the participants and therefore are measured once and expected not to change (e.g., participant race and sex) along with data that are characteristics of participants at a particular moment in time and therefore are measured multiple times (e.g., a participant's scores on a depression inventory). Storage of data that apply at different levels in separate datasets eliminates dataset redundancy and allows for streamlined correction of dataset errors (because any errors that are discovered only have to be corrected in one place rather than multiple). For these reasons, we prefer that processed data that apply to different units of analysis are stored in separate datasets. However, this is not a strict rule, and we will allow exceptions to it in special cases. When processed data are stored in separate datasets, each dataset should contain appropriate ID variables (participant IDs, lab IDs, etc) that allow the separate datasets to be merged for analysis.

The values stored in the processed dataset should be as descriptive as possible to avoid misinterpretations during analysis. For example, we prefer participant sex to be recorded using the text values "male" and "female" rather than " 0 " and " 1 ". We prefer the use of "NA" to indicate missing values, especially in processed datasets.

\section{Codebooks}

A dataset's codebook is the primary means of documenting its contents. Codebooks come in different styles; for example, see here for a style guide on the Open Science Framework. PSA codebooks should consist of a .csv (or other machine readable and accessible) file containing, at a minimum, the following columns:

- variable_name: The name of the variable in question, as it appears in the dataset.

- variable_description: A written description of the variable. For survey questions, the actual text of the question.

- variable_values: The values that the variable can take. If the values that are recorded in the dataset are different from the values the participant sees (e.g., if a participant responded to the labels "strongly agree", "agree", etc, but these were recorded as numbers in the dataset), document these differences here. 
The PSA prefers that the names it uses for its variables are harmonized across datasets to allow for easy merging of variables across different projects. For this reason, we track the variable names used in different datasets in a common database. We do not yet have an ontology that we use to generate variable names, though we hope to develop one in the future. For the time being, source and raw will use the names given by the proposing authors; the project's data manager (a person selected by the PSA's Assistant Director of Data who has with primary responsibility for managing a given project's data) may suggest different names in the processed dataset to facilitate name harmonization.

\section{Confidentiality issues}

In their original form, data are likely to contain identifying information. For example, pictures and videos are likely to contain faces, which make participants identifiable. Source and raw data that contain identifying information will generally require stronger protections for data security.

However, even if no single piece of data makes participants identifiable, a combination of variables may (Narayanan, \& Shmatikov, 2008; El Emam, Jonker, Arbuckle, \& Malin, 2011). For example, knowledge of the location of data collection along with knowledge of membership within a rare group (for example, a rare gender category) could make a participant identifiable. Identifiability is of greatest concern when some of the data collected in a study is sensitive and/or puts the participant at risk if it is revealed to the general public.

Proposing teams should elaborate on any confidentiality issues that affect how data are secured during collection, and how source, raw, and processed datasets are shared after collection. If necessary, the proposing team can propose to either strip identifying information from the raw datasets prior to deposition or pool across larger demographic units to make identification less likely. Alternatively, the proposing team can propose to limit public access to source and raw datasets by implementing an approval procedure that secondary analysts should follow to gain access. We strongly prefer that processed datasets are in a freely shareable format so that the analyses to facilitate easy reproduction of the analyses reported in the research documents that rely on those datasets. In cases where datasets cannot be shared, any code used to help transform the data from one state to another (source to raw, raw to processed) should be written in such a way that it can be shared.

The PSA will generally only limit access to datasets when absolutely necessary and as required by local regulations (e.g., HIPAA in the United States, GDPR in the European Union). 


\section{Data Repository}

All datasets and codebooks associated with a given proposer's project will be deposited on a common project on the Open Science Framework. The data manager has ultimate responsibility for ensuring that the data repository has the structure recommended below. 


\section{Recommended Repository Structure}

PSA uses an OSF repository by default, but other independent repositories, such as Databrary, can be selected on a case-by-case basis. PSA projects should follow the following structure:

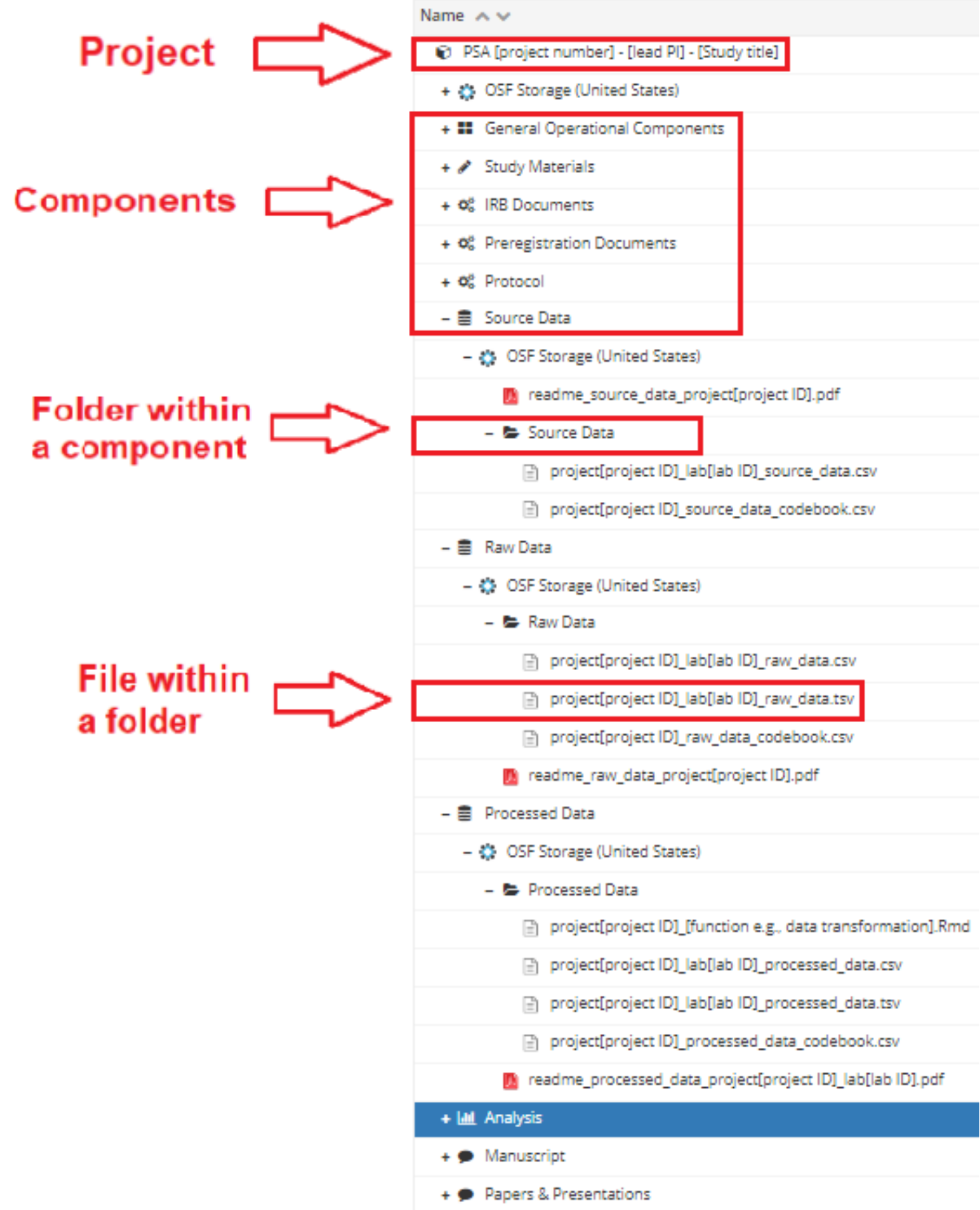

Figure 2. Recommended directory structure 
Components should be used for major parts of the project (e.g., Study Materials, Raw Data etc.) as components can have their own privacy setting.

Folders should be used to organize the content within the Components (for an overview of how to do this on OSF, see this guide).

- Codebooks should be stored in the same component as their dataset.

- Syntax that gets Raw Data into Processed Data should be stored in the same component as the Processed Data. If needed or desired, proposing teams can link to external repositories that host syntax, such as GitHub.

- Add wiki when further explanation is necessary.

\section{Nomenclature}

\begin{tabular}{|l|l|}
\hline Project title & [project ID] - [lead PI] - [Study title] \\
\hline Dataset file name & $\begin{array}{l}\text { [project ID]_lab[lab ID]_source_data.csv } \\
\text { [project ID]_lab[lab ID]_raw_data.csv } \\
\text { [project ID]_lab[lab ID]_raw_data.tsv } \\
\text { [project ID]_lab[lab ID]_processed_data.csv } \\
\text { [project ID]_lab[lab ID]_processed_data.tsv }\end{array}$ \\
\hline Codebook file title & $\begin{array}{l}\text { [project ID]_raw_data_codebook.csv } \\
\text { [project ID]_raw_data_description.json }\end{array}$ \\
\hline Syntax file title & [project ID] [function e.g., data transformation].Rmd \\
\hline
\end{tabular}

\section{OSF project template}

The PSA OSF project template can be accessed from here: https://osf.io/kx6e4/. One can duplicate it by clicking on the fork button and selecting 'duplicate' in the top right of the screen.

\section{Access settings}

Everyone on a proposing team is granted access rights unless the proposing team agrees otherwise. These people are granted "owner" rights to the whole OSF project.

The project's data manager should control the project's access settings. The data manager should grant edit rights to people on an as-needed basis and should generally revoke a person's edit rights once they have completed their role in that project's data management process. 


\section{Meta-Data}

Meta-data are data that provide information about the process that led to the collection of the primary study data. Although meta-data may not bear directly on a study's primary question of interest, they can be of immense value for secondary analysis, especially for meta-science projects dedicated to understanding the processes that lead to more or less credible evidence. The Psychological Science Accelerator is committed to collecting meta-data that may help advance this understanding.

Just as primary data can have a complex structure, meta-data can have a nested structure in that different meta-data variables can apply to different units of analysis. Table 4 lays out a few typical units of analysis.

\begin{tabular}{l|l|l} 
Level & Description & Examples \\
\hline Project-level & Applies to the entire research project & $\begin{array}{l}\text { Names of the project proposers } \\
\text { Software used for data collection }\end{array}$ \\
\hline Lab-level & $\begin{array}{l}\text { Applies to the participating lab that obtained a particular } \\
\text { set of responses }\end{array}$ & $\begin{array}{l}\text { Geographic location of the lab } \\
\text { Institutional affiliation of the lab }\end{array}$ \\
\hline Session-level & $\begin{array}{l}\text { Applies to a particular data collection session in which a } \\
\text { group of people gave their responses }\end{array}$ & $\begin{array}{l}\text { Session start } \\
\text { Session end }\end{array}$ \\
\hline Participant-level & $\begin{array}{l}\text { Applies to a particular person with whom a set of } \\
\text { responses are associated }\end{array}$ & $\begin{array}{l}\text { Participant race } \\
\text { Participant gender }\end{array}$ \\
\hline Response-level & Applies to a particular response & Time the response was given
\end{tabular}

Table 4. Typical units of analysis to which meta-data can apply.

Project-leve/ meta-data should generally be stored on a project's OSF page (see here for more details) and/or the .json file that is stored alongside a project's processed datasets (see below).

Lab-level meta-data will generally come from the lab profiles maintained by the Community Building and Network Expansion Committee.

Session-level, participant-level, and response-level meta-data should be collected as part of the collection of a project's primary data. We encourage proposing labs to be thoughtful about what meta-data will provide insight into the process used to generate a project's primary data.

The PSA has some minimum standards for meta-data that should be collected in every project:

- The project's project ID.

- The lab ID for the lab that obtained a particular group of responses. Note that this ID identifies the whole lab rather than a particular person, such as a PI, to allow for multimember labs. The lab ID is critical for allowing merging of project data with data collected on PSA member labs.

- Session start date, using ISO format for processed datasets, and, if possible, for raw datasets. If confidentiality considerations permit, we prefer datasets to also contain 
session start times and session end times. For sensitive datasets, adding this information may bring too much risk to the participants due to the possibility of reidentification. Whether it is worthwhile to add session start and end times should be evaluated on a project-by-project basis.

- A participant ID that distinguishes between participants recruited by a particular lab.

We also recommend, but do not require, that laboratory notebooks be stored as lab-level meta-data. See the "Data Integrity Checks" section for more information.

To adhere to the Psych-DS dataset specification, some meta-data should be stored in a .json file called "[project_id]_[dataset name]_description.json" should be archived alongside the project's processed dataset. A .json file is a machine-readable structured text file that contains basic information about a dataset. It can be thought of as a machine-friendly codebook. The project's data manager will prepare this .json file. 


\section{Data Integrity Checks}

Data integrity checks are designed to ensure adherence to the principles that guide our data management process, namely ethics, security, accuracy, usability, and transparency. Data integrity focuses particularly on maximizing accuracy and usability. In general, data integrity checks are divided into two types:

- Issues flagged by each site during data collection

- Checks conducted during data processing

\section{Issues flagged during data collection}

In general, we encourage the teams that propose our projects to use a centralized data means of data collection so that the data management process will also be centralized. However, some projects may require the presence of an experimenter during data collection - for example, it may not be practical to conduct a study of interpersonal interactions over the internet. In these cases, experimenter notes play an important role in ensuring the integrity of data.

For these types of experiments, we encourage each data collection site to keep a detailed laboratory notebook of events that may be relevant to the validity, accuracy, and completeness of the data (see this link for an example from Heather Urry's lab). This includes events or errors that may have prevented some data from being recorded. Here are some examples of events that should be recorded in the laboratory notebook:

- An equipment failure prevents the recording of a portion of the data.

- The participant is visibly hungover and is therefore not attentive during the experiment.

- The participant is texting on their phone throughout the experiment and therefore is not paying attention.

The specific events that should be recorded may vary from project to project. These events will be reviewed by the Data Management Committee during the data processing stage.

Laboratory notebooks are not required to be kept as online records. However, if these notebooks are kept as written records, the notes should be archived alongside the primary lab data, either as a column of session-level meta-data or as a scanned document that is archived in the project's data repository.

\section{Checks during data processing}

Although not all projects will involve the in-person presence of an experimenter, all projects will involve some data integrity checks during and after the data processing stage. These checks are typically spearheaded by the project's data manager, who is selected by the PSA's Assistant Director of Data. The general purpose of these checks is to identify and correct 
issues that occurred in the process of acquiring source data, transforming source data to raw, and raw data to processed. The specific procedures used will vary from project to project. However, here is a list that may be helpful:

- Examination that all variables (including ID variables) have appropriate values (i.e., that they fall within the appropriate range of possible responses, e.g., a 7-point Likert scale only has responses from 1-7)

- Examination that all variables have appropriate means, standard deviations, distributions, and/or additional summary statistics (i.e., frequencies and crosstabulations)

- Examination of observations that have highly unusual values.

- Examination of whether each participant and lab ID is associated with an appropriate number of observations

- Examination of the data processing script for errors, warnings, and bugs

- Verification that any datasets that are made publicly available don't contain information that they shouldn't (for example, data that render participants identifiable)

In and of themselves, these procedures usually cannot identify the causes of the anomalous data patterns. However, they can help identify potential causes that may warrant further investigation.

Errors identified during the data integrity check should be documented, usually in the script that transforms the raw data into the processed data. Errors diagnosed during the data integrity check should be reviewed by at least one person aside from the initial error identifier.

\section{Exclusion of observations from analysis}

Decisions about the inclusion or exclusion of observations should be primarily based on preregistered criteria for exclusion. Exclusions that occur based on non-preregistered criteria should be clearly documented and justified. Some of these post-hoc exclusions may be based on the review of the laboratory notebooks. These post-hoc exclusions should be reviewed by at least one person, selected by the data manager, who is outside the proposing team blind to the critical study variables. These decisions should also always be made in as uniform a manner as possible and can occur in one step (i.e., the proposing team can submit a full list of exclusion decisions that is reviewed in one pass).

The raw data should be unaffected by decisions of exclusion from analysis. That is, excluded cases should not be deleted from the raw data. Rather, they should be flagged in an extra dataset column as excluded from the primary analyses. We recommend this flagging occur in the processed dataset to facilitate easier checks on the robustness of the primary analyses (e.g., analyses with and without the excluded observations). We recommend tracking which observations are to be excluded with the following dataset columns: 'excluded_from_analysis' [possible values: yes, no], and 'reason_for_exclusion' [text field with a clear description for the reason for exclusion]. 


\section{Data Release}

The process of the release of PSA data will start when any public-facing document that presents analyses based on the data is scheduled for public distribution - for example, a preprint is posted on a public server or a manuscript is accepted for publication. Research products based on secondary analysis of a project's dataset should never be made public until all a project's non-confidential data are public.

The data release process is governed by a data release plan, a subsection of the data management plan which is drafted prior to the posting of any preprint or other public-facing research document by the proposing author team, and, if needed, the project's data manager. The data release plan is also subject to the approval of the Assistant Directors of the D\&M Committee, who will review it and offer feedback if necessary. At a minimum, the data release plan specifies the license under which data are shared, whether the data are subject to full release or phased release, the timing of the stages of release, and whether and how outside researchers can gain access to confidential parts of the data.

\section{License}

The license governs the legal aspects of data sharing. Licenses range from ones that place few restrictions on the reuse of a work (i.e., the sharer does not retain the right to exercise control over how the work is reused) to ones that place many restrictions on reuse. The family of licenses that places the fewest restrictions on reuse is called Creative Commons.

The PSA wishes for its works to be as freely shareable as possible and thus strongly encourages the use of Creative Commons licenses. In particular, the PSA recommends (but does not require) the use of the "No Rights Reserved" Creative Commons (CCO) license, which places the least restrictions on re-use.

Note that the choice of a "No Rights Reserved" license does not mean that the work is not subject to academic citation norms. The "No Rights Reserved" license only governs the legal aspects of re-use.

Most repositories allow the user to choose the license under which the contents are shared. On the Open Science Framework, the place to choose the license is shown in Figure 2.

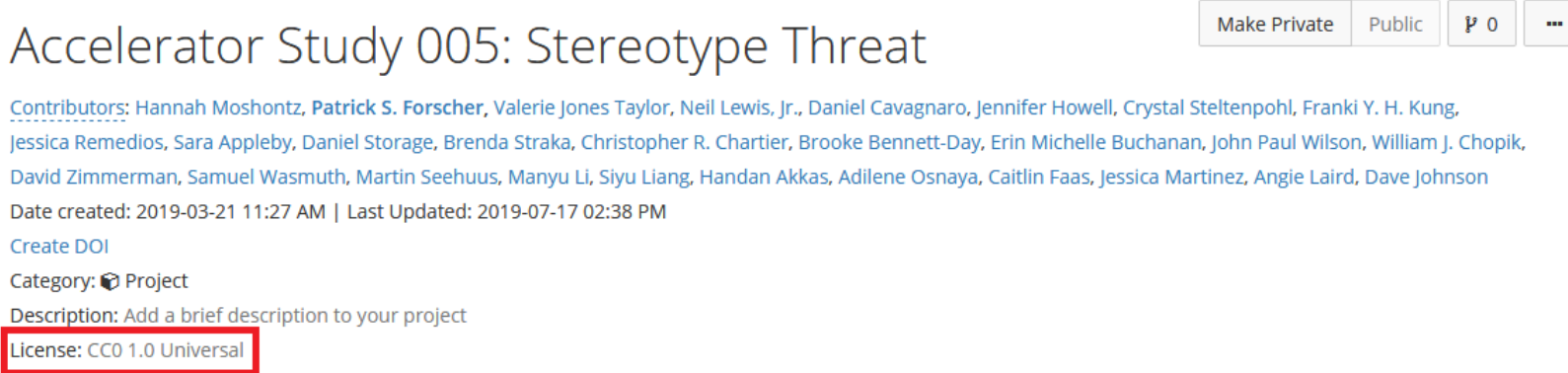

Figure 2. Selecting a CCO license for a PSA project using the Open Science Framework. 


\section{Full Release}

In the case of full release, non-confidential data are deposited in a public repository and the confidential data in a private one, all in accordance with the policies outlined elsewhere in this document. Optionally, non-confidential data can be born open - that is, publicly accessible from the very time they are recorded (Rouder, 2016).

Once the non-confidential data are made public, the data manager for the project should publicly announce the dataset release to maximize the data's value to the scientific community.

\section{Phased Release}

In contrast to a full release, phased release allows for release of the data in separate segments. Most typically there will be two: an exploratory segment $(\sim 1 / 3$ of the data) to facilitate hypothesis generation and a confirmatory segment $(\sim 2 / 3$ of the data) to facilitate hypothesis testing. By clearly separating exploration from confirmation, this release schedule should allow for maximally informative secondary data use.

Another benefit of phased release occurs in situations where the proposing team has opted to submit a Registered Report. In such cases, the Director or Associate Directors can contact the journal hosting the Registered Report to ask the journal to accept commentaries on the Registered Report using secondary analyses prepared as part of the data release plan. The proposing team should contact the journal immediately after their in-principle acceptance so that information about these commentaries can be incorporated into the data release announcements. Because of the benefits of phased release, we recommend that proposing teams use this release structure when possible.

Phased data release plans are necessarily more complicated than immediate ones. Below, we elaborate on the extra required elements beyond what we expect of immediate release plans.

\section{Announcement of Data Release}

The first stage of phased release is a public announcement of the dates for the release of the exploratory and confirmatory segments of the data. Although the exact timing of this announcement can vary, we suggest it occur approximately twelve months before the release of the confirmatory segment, possibly at the time of an in-principle acceptance of a Registered Report for the project. The announcement should contain a link to a codebook and a sample containing, for example, simulated data or pilot data. The sample dataset will follow the structure, formatting, and conventions established in the data management plan. This will also allow people outside the proposing team to inspect the data structure and variable names for possible errors and to prepare any secondary analysis plans of their own.

If the data release plan entails commentaries on the paper, the invitation for commentaries should also go in the data release announcement. 


\section{Release of the Exploratory Segment}

The purpose of the exploratory segment is to allow researchers to assess patterns of the data and prepare preregistered analyses for the confirmatory segment. The specific cases that are go in the exploratory segment (as opposed to the confirmatory segment) should be chosen at random, using a sampling method that respects the clustering of participants within labs.

We recommend that enough data go into the exploratory segment to ensure that any discovered patterns are relatively stable, but not much as to render statistical tests in the confirmatory segment uninformative. In most situations, we believe this will amount to between $1 / 3$ and $1 / 2$ of the dataset.

The time lag between the release of the exploratory and confirmatory segments can vary from project to project, but this lag should be long enough to give researchers (especially those outside of the PSA network) sufficient time to discover the statistical patterns they wish to test in the confirmatory segment. In most cases, we believe that a lag of six months between the release of the exploratory and confirmatory segments will be sufficient. The exact time of release should occur at a randomly selected top of the hour between 12:00AM and 11:00PM Universal Coordinated Time (UTC).

\section{Release of the Confirmatory Segment}

The purpose of this segment is to allow hypothesis testing using preregistered scripts that are written using the exploratory segment. Any data not included in the exploratory segment should be included in the confirmatory segment.

If the proposing team has opted to allow commentaries on their manuscript to be published alongside the main manuscript, the timing of this release should occur such that the commentary authors have sufficient time to incorporate preregistered analyses of the confirmatory segment in their commentaries. The specific time of the release of the confirmatory segment should occur at a new randomly selected top of the hour between 12:00AM and 11:00PM Universal Coordinated Time (UTC). 


\section{References}

El Emam, K., Jonker, E., Arbuckle, L., \& Malin, B. (2011). A Systematic Review of ReIdentification Attacks on Health Data. PLoS ONE, 6(12), e28071. https://doi.org/10.1371/journal.pone.0028071

Narayanan, A., \& Shmatikov, V. (2008). Robust De-anonymization of Large Sparse Datasets. In 2008 IEEE Symposium on Security and Privacy (sp 2008) (pp. 111-125). Oakland, CA, USA: IEEE. https://doi.org/10.1109/SP.2008.33

Rouder, J. N. (2016). The what, why, and how of born-open data. Behavior Research Methods, 48(3), 1062-1069. https://doi.org/10.3758/s13428-015-0630-z

Wickham, H. (2014). Tidy Data. Journal of Statistical Software, 59(10). https://doi.org/10.18637/jss.v059.i10

Wilkinson, M. D., Dumontier, M., Aalbersberg, I. J., Appleton, G., Axton, M., Baak, A., ... Mons, B. (2016). The FAIR Guiding Principles for scientific data management and stewardship. Scientific Data, 3, 160018. https://doi.org/10.1038/sdata.2016.18 